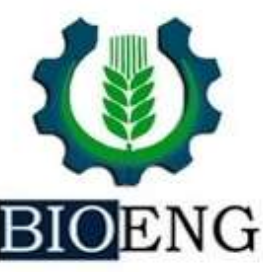

\title{
ADUBAÇÃO NITROGENADA NA IMPLANTAÇÃO DE Urochloa brizantha cv. XARAÉS NO CERRADO: Características nutricionais - Parte 2
}

\author{
BIOENG \\ J. M. K. Santini ${ }^{1}$, A. Perin ${ }^{2}$, D. N. Coaguila ${ }^{1}$, M. Valderrama ${ }^{3}$, \\ F. S. Galindo ${ }^{1}$, C. G. dos Santos ${ }^{2}$, V. M. Silva ${ }^{1}$, S. Buzetti $^{1 *}$ \\ ${ }^{1}$ UNESP - Univ Estadual Paulista, Faculdade de Engenharia de Ilha Solteira, Ilha Solteira, \\ SP, Brasil \\ ${ }^{2}$ IFG - Instituto Federal Goiano,Campus Rio Verde, Rio Verde, GO, Brasil \\ ${ }^{3}$ MASTER AGRO Produtos Agrícolas Ltda., Avaré, SP, Brasil
}

Article history: Received 04 May 2016; Received in revised form 10 June 2016; Accepted 13 June 2016; Available online 30 July 2016.

\section{RESUMO}

Entre os fatores agrícolas, a adubação tem proporcionado grandes avanços na produtividade e qualidade de pastagens, sendo que o nitrogênio $(\mathrm{N})$ merece destaque por sua grande extração e aumento da produtividade de gramíneas, entretanto, sofre influência do meio e pode ser perdido se mal manejado. Para minimizar tal problema, uma alternativa apontada como promissora é o uso de fertilizantes de eficiência aumentada. Dessa forma, objetivou-se com o trabalho avaliar o efeito de fontes e doses de $\mathrm{N}$ na implantação do Urochloa brizantha cv. Xaraés (capim-xaraés), em cultivo nas condições do Cerrado. O experimento foi conduzido em área experimental no município de Rio Verde - GO, Brasil, com delineamento em blocos ao acaso com quatro repetições, em esquema fatorial de $3 \times 4$. Sendo três fontes de $\mathrm{N}$ (ureia convencional, ureia de eficiência aumentada e nitrato de amônio); e quatro doses de $\mathrm{N}\left(0,20,40\right.$ e $80 \mathrm{~kg} \mathrm{~N} \mathrm{ha}^{-1}$ corte $\left.^{-1}\right)$. O uso da ureia convencional fez-se como a melhor alternativa para a cultura do capim-xaraés, a ureia revestida não foi considerada de maior eficiência. A dose de nitrogênio recomendada é de $80 \mathrm{~kg} \mathrm{ha}^{-1}$ por corte, em vista da melhoria nutricional da matéria seca da parte aérea.

Palavras-chave: Brachiaria brizantha, fertilizante de eficiência aumentada, manejo de pastagens.

\section{NITROGEN FERTILIZATION IN IMPLEMENTATION OF Urochloa Brizantha cv. Xaraés IN BRAZILIAN SAVANNA: Nutritional characteristics - Part 2}

\begin{abstract}
Among the productivity-enhancing factors, fertilization has provided great advances in productivity and quality of pastures, and the nitrogen $(\mathrm{N})$ is emphasized for its large extraction and increasing the productivity of grasses, however, it is influenced by the environment and can be lost if mismanagemented. To minimize this problem, an alternative that has been identified as promising is the use of enhanced efficiency fertilizers. Thus, the aim of the work was to evaluate the effect of sources and doses of $\mathrm{N}$ in the implementation of Urochloa brizantha cv. Xaraés (xaraés-grass), in cultivation in the Cerrado conditions. The experiment was conducted at the experimental area in Rio Verde - GO, Brazil, the experimental design was a randomized block with four replications, in a factorial scheme of

* $\underline{\text { sbuzetti@agr.feis.unesp.br }}$
\end{abstract}


$3 \times 4$. With three sources of $\mathrm{N}$ (conventional urea, enhanced efficiency urea and ammonium nitrate); and four $\mathrm{N}$ doses $\left(0,20,40\right.$ and $\left.80 \mathrm{~kg} \mathrm{~N} \mathrm{ha}^{-1} \mathrm{clip}^{-1}\right)$. The use of conventional urea was best alternative for growing xaraés-grass, the enhanced urea was not deemed greater efficiency. The recommended dose of nitrogen is $80 \mathrm{~kg} \mathrm{ha}^{-1}$ by clipping, considering the nutritional improvement of dry matter shoot.

Keywords: Brachiaria brizantha, enhanced efficiency fertilizers, pasture management.

\section{INTRODUÇÃO}

A pecuária brasileira é uma das principais atividades econômicas do país, e de grande importância no mercado internacional. De acordo com o USDA (2014), o Brasil é um dos principais produtores de carne bovina e o maior exportador, entretanto, para tal, a pecuária brasileira ocupa $48 \%$ da área rural do país (IBGE, 2006), mas tem apresentado baixos índices zootécnicos, principalmente relacionados a manejo nutricional $\mathrm{e}$ sanitário inadequado e com o baixo potencial genético dos animais (HOFFMANN et al., 2014)

Visando maiores produtividades, devido à baixa eficácia da pecuária brasileira, o estudo de nutrição das forrageiras tem chamado atenção da comunidade cientifica, de acordo com FAGUNDES et al. (2006), o potencial de produção de planta forrageira é determinado geneticamente, porém, para que este potencial seja alcançado, são necessárias condições do meio e manejos adequados. Seguramente, entre as condições do meio e manejo, a baixa disponibilidade de nutrientes e a incorreta adubação, são fatores que mais interferem na produtividade e na qualidade da forrageira. Também, os autores relatam que $o$ fornecimento de nutrientes, em quantidades e proporções adequadas, particularmente o $\mathrm{N}$, assume grande importância no processo produtivo de pastagens.

$\mathrm{O} \mathrm{N}$ é um dos nutrientes requeridos em maiores quantidades pelas gramíneas forrageiras, sendo elemento estrutural e participando de vários processos metabólicos (SOUZA \& FERNANDES, 2006). Vários autores têm observado influências positivas da adubação nitrogenada no capim-xaraés (FAGUNDES et al., 2006; CABRAL et al., 2012; COSTA et al., 2013), mas, a eficiência da aplicação é, isoladamente, o fator que mais contribui para a viabilidade agrícola, já que o N, após a aplicação, passa por influências do meio, podendo ser perdido por lixiviação, alcançando o lençol; ou por volatilização, na forma de $\mathrm{NH}_{3}$.

Manejos inadequados e com baixa eficiência, tem sido demasiadamente criticados, principalmente quando podem culminar em degradação ambiental. Assim, devido à responsividade do capim-xaraés, ao crescente uso de adubos e as altas perdas de $\mathrm{N}$, torna-se necessária a utilização de manejos mais eficientes, evitando assim, gastos desnecessários e obtendo-se maiores produtividades.

Para reduzir as perdas oriundas dos fertilizantes nitrogenados, o uso de fertilizantes de eficiência aumentada é mencionado como boa opção. Os fertilizantes de eficiência aumentada possuem revestimentos físicos e/ou químicos que propiciam condições de controle e contribuem para a sincronia de liberação de nutrientes, de acordo com as necessidades nutricionais das plantas, podendo resultar em maiores produtividades (VIEIRA \& TEIXEIRA 2004). Além de reduzir as perdas, estes fertilizantes podem trazer diminuição aos custos de produção e reduzir os impactos ambientais (VALDERRAMA et al., 2009).

Inerente ao supracitado, acredita-se que as fontes nitrogenadas e o uso de doses maiores de $\mathrm{N}$, acarretarão maior absorção de nutrientes, que vai gerar maiores produtividades, necessitando maior cuidado na implantação da forrageira. 
Vale ressaltar que há carência de estudos sobre uso de fertilizantes de eficiência aumentada no Cerrado brasileiro, principalmente quando se trata de adubação de pastagens. Além de que, estudos inerentes à implantação de pastagens são de grande valia, pois a

\section{MATERIAL E MÉTODOS}

O experimento foi conduzido na Estação Experimental do Instituto Federal de Ciência e Tecnologia Goiano - câmpus Rio Verde, GO, com localização geográfica $17^{\circ} 48^{\prime}$ de latitude Sul e $50^{\circ} 54^{\prime}$ longitudes Oeste, com altitude de $748 \mathrm{~m}$, em uma gleba de $1.400 \mathrm{~m}^{2}$. A gleba possui solo classificado como Latossolo Vermelho distroférrico (EMBRAPA, 2013), com histórico de uso agrícola em sistema de rotação de culturas, com predominância da soja na primeira safra, milho na segunda safra.

A precipitação pluvial durante $\mathrm{o}$ experimento foi de: outubro (2012): 113 $\mathrm{mm}$; novembro (2012): $335 \mathrm{~mm}$; dezembro (2012): $127 \mathrm{~mm}$; janeiro (2013): $475 \mathrm{~mm}$; fevereiro (2013): $270 \mathrm{~mm}$; e março (2013): $552 \mathrm{~mm}$, totalizando $1872 \mathrm{~mm}$, e a temperatura máxima e mínima média foi de $30,8 \pm 1,3 ;$ e $19,6 \pm 0,1$, respectivamente.

Antes da instalação do experimento, foi realizada análise de fertilidade e textura do solo, em profundidade de 0-0,2 m, obtendo-se os seguintes resultados: $\mathrm{pH}$ $\left(\mathrm{CaCl}_{2}\right)=5,3 ;$ M.O. $=33 \mathrm{~g} \mathrm{dm}^{-3} ; \mathrm{P}$ (Resina) $=29 \mathrm{mg} \mathrm{dm}^{-3} ; \mathrm{K}=5,9 \mathrm{mmol}_{\mathrm{c}} \mathrm{dm}^{-}$ 3. $\mathrm{Ca}=40 \mathrm{mmol}_{\mathrm{c}} \mathrm{dm}^{-3} ; \mathrm{Mg}=16 \mathrm{mmol}_{\mathrm{c}}$ $\mathrm{dm}^{-3} ; \mathrm{Al}=0 \mathrm{mmol}_{\mathrm{c}} \mathrm{dm}^{-3} ; \mathrm{H}+\mathrm{Al}=22$ $\mathrm{mmol}_{\mathrm{c}} \mathrm{dm}^{-3}$; S.B. $=61,9 \mathrm{mmol}_{\mathrm{c}} \mathrm{dm}^{-3}$; CTC $=83,9 \mathrm{mmol}_{\mathrm{c}} \mathrm{dm}^{-3} ; \mathrm{V} \%=74 ; \mathrm{B}=0,29 \mathrm{mg}$ $\mathrm{dm}^{-3} ; \mathrm{Cu}=3,4 \mathrm{mg} \mathrm{dm}^{-3} ; \mathrm{Fe}=14 \mathrm{mg} \mathrm{dm}^{-3}$; $\mathrm{Mn}=30,6 \mathrm{mg} \mathrm{dm}^{-3} ; \mathrm{Zn}=2,6 \mathrm{mg} \mathrm{dm}^{-3} \mathrm{e}$ textura média (331 $\mathrm{g} \mathrm{kg}^{-1}$ de argila, $167 \mathrm{~g}$ $\mathrm{kg}^{-1}$ de silte e $502 \mathrm{~g} \mathrm{~kg}^{-1}$ de areia).

Os tratamentos foram distribuídos em blocos ao acaso com quatro repetições, em esquema fatorial de $3 \times 4$. O primeiro fator foram fontes de fertilizantes nitrogenados (ureia convencional, ureia revestida e nitrato de amônio) e o segundo implantação incorreta e o fator inicial para acarretar a degradação do pasto. Dessa forma, buscou-se avaliar o efeito de fontes e doses de $\mathrm{N}$, à lanço, na implantação do Urochloa brizantha cv. Xaraés (capimxaraés), na concentração de nutrientes em cultivo nas condições do Cerrado.

fator foram doses de nitrogênio em cobertura $\left(0,20,40\right.$ e $80 \mathrm{~kg} \mathrm{~N} \mathrm{ha}^{-1}$, após cada corte). Cada parcela possuiu 6 metros de comprimento por 3,15 metros de largura. Considerada como área útil da parcela 5 metros por 2,15 metros centrais.

No dia 10 de outubro de 2012, após o preparo do solo, foi distribuído o equivalente a $7 \mathrm{~kg} \mathrm{ha}^{-1}$ de sementes puras viáveis a lanço. Junto à semeadura foram $90 \mathrm{~kg} \mathrm{ha}^{-1}$ de $\mathrm{P}_{2} \mathrm{O}_{5}$ (superfosfato triplo) e $100 \mathrm{~kg} \mathrm{ha}^{-1}$ de $\mathrm{K}_{2} \mathrm{O}(\mathrm{KCl})$, conforme recomendação de Sousa e Lobato (2004).

Durante todo o período do experimento, foram realizados controles de plantas invasoras de forma manual e com o uso de herbicida seletivo do Grupo Químico Triazina (Atrazine), na dose de $1,5 \mathrm{~kg} \mathrm{ha}^{-1}$ do ingrediente ativo e volume de calda equivalente $200 \mathrm{~L} \mathrm{ha}^{-1}$.

A adubação nitrogenada foi realizada a lanço, com as doses de 0,20, 40 e $80 \mathrm{~kg}$ $\mathrm{N}$ ha $^{-1}$ corte $^{-1}$ em seus respectivos tratamentos, nos três cortes realizados (totalizando 0, 60, 120 e $240 \mathrm{~kg} \mathrm{~N} \mathrm{ha}^{-1}$ no período experimental). A primeira aplicação dos fertilizantes foi realizada 50 dias após emergência (DAE) (15/12/2012) e no dia 14/01/2013 foi realizado o primeiro corte. Os cortes subsequentes foram realizados em 14/02 e 15/03/2013, efetuando a adubação de cobertura posterior a cada corte, exceto ao último.

As amostragens foram realizadas aleatoriamente na área útil da parcela, com corte à $15 \mathrm{~cm}$ de altura do solo, utilizando demarcador de $0,5 \mathrm{~m}^{2}$. Após a coleta das amostras, o restante do capim era ceifado com roçadora mecânica e removido das parcelas.

A forragem após colhida foi embalada em sacos de papel e colocadas 
para secar em estufa de circulação de ar forçada, com temperatura de $65^{\circ} \mathrm{C}$, por 72 horas. Com as amostras secas, realizou-se a pesagem, e subsequente, os dados foram convertidos em $\mathrm{kg} \mathrm{ha}{ }^{-1}$. Em seguida, as amostras produzidas na área representativa foram moídas em moinho tipo Wiley equipado com peneira com crivos de 1 $\mathrm{mm}$, e armazenadas, no aguardo das análises nutricionais.

Foram realizadas as análises químicas foliares, como variável resposta, para determinação dos teores de N, P, K, $\mathrm{Ca}, \mathrm{Mg}, \mathrm{S}, \mathrm{B}, \mathrm{Cu}, \mathrm{Fe}, \mathrm{Mn}$ e $\mathrm{Zn}$, conforme metodologia descrita por MALAVOLTA et al. (1997).

Inicialmente, com os dados, realizouse o teste de Kolmogorov - Smirnov ( $\mathrm{p}<$ 0,05), buscando avaliar a homocedasticidade, quando não alcançada, procedeu a transformação de dados,

\section{RESULTADOS E DISCUSSÃO}

No presente estudo não se verificou interação entre as fontes e doses de $\mathrm{N}$ para as variáveis avaliadas $(\mathrm{p} \geq 0,05)$. A concentração de $\mathrm{N}$ foliar não diferiu entre as fontes estudadas, já para as doses houve aumentos lineares nos três cortes (Tabela utilizando a equação $(x+0,5)^{0,5}$. Os dados foram submetidos à análise de variância utilizando o programa estatístico SISVAR (FERREIRA, 2003). Para as variáveis qualitativas que diferiram significativamente, as médias foram comparadas pelo teste Tukey à 5 e $1 \%$ de probabilidade, já paras as variáveis quantitativas foram realizadas regressões polinomiais à 5 e $1 \%$.

Foram realizadas análises de correlações de Pearson ( $p<0,05 ; 0,01$ ) entre as concentrações nutricionais, da parte aérea, do capim-xaraés, inerentes ao período experimental total (três cortes). $\mathrm{Na}$ avaliação de teores médios nutricionais, os dados foram submetidos ao teste não paramétrico de Kruskal-Wallis, ao nível de $5 \%$ de probabilidade, e para comparação de médias os dados foram submetidos ao teste de Mann-Whitney a 5\%.

Tabela 1. Concentração nutricional e exportação de nitrogênio, fosforo e potássio da parte aérea do capim-xaraés, em função de fontes e doses nitrogenadas em cobertura

\begin{tabular}{|c|c|c|c|c|c|c|c|c|c|}
\hline \multicolumn{10}{|c|}{ Concentração nutricional foliar $\left(\mathrm{g} \mathrm{kg}^{-1}\right)$} \\
\hline \multirow{3}{*}{ Fontes } & \multicolumn{3}{|c|}{$\mathrm{N}$} & \multicolumn{3}{|c|}{$\mathrm{P}$} & \multicolumn{3}{|c|}{$\mathrm{K}$} \\
\hline & \multicolumn{3}{|c|}{ Corte } & \multicolumn{3}{|c|}{ Corte } & \multicolumn{3}{|c|}{ Corte } \\
\hline & $1^{\mathrm{o}}$ & $2^{\circ}$ & $3^{\circ}$ & $1^{\mathrm{o}}$ & $2^{\circ}$ & $3^{\circ}$ & $1^{\mathrm{o}}$ & $2^{\circ}$ & $3^{\circ}$ \\
\hline $\mathrm{U}$ & $13,2^{\mathrm{ns}}$ & $20,8^{\mathrm{ns}}$ & $18,4^{\mathrm{ns}}$ & $2,3^{\mathrm{ns}}$ & $3,2^{\mathrm{ns}}$ & $3,7^{\mathrm{ns}}$ & $25^{\mathrm{ns}}$ & $22 b$ & $22^{\mathrm{ns}}$ \\
\hline UR & 12,4 & 20,4 & 17,2 & 2,3 & 3,6 & 3,8 & 24 & $26 \mathrm{a}$ & 20 \\
\hline NA & 13,0 & 24,1 & 18,5 & 2,3 & 3,4 & 3,6 & 24 & $24 \mathrm{ab}$ & 22 \\
\hline $\begin{array}{c}\text { Doses (kg } \\
\left.\mathrm{ha}^{-1}\right)\end{array}$ & $1^{\mathrm{o}}$ & $2^{\circ}$ & $3^{\circ}$ & $1^{\mathrm{o}}$ & $2^{o}$ & $3^{\circ}$ & $1^{o}$ & $2^{\circ}$ & $3^{\circ}$ \\
\hline 0 & $10,5^{1}$ & $19,0^{2}$ & $13,6^{3}$ & $2,2^{\mathrm{ns}}$ & $3,7^{\mathrm{ns}}$ & $3,9^{\mathrm{ns}}$ & $22^{4}$ & $23^{5}$ & $17^{6}$ \\
\hline 20 & 12,7 & 19,9 & 16,5 & 2,3 & 3,5 & 3,9 & 24 & 24 & 20 \\
\hline 40 & 13,2 & 20,6 & 19,5 & 2,3 & 3,2 & 3,3 & 27 & 24 & 23 \\
\hline 80 & 15,3 & 27,5 & 22,4 & 2,4 & 3,4 & 3,6 & 25 & 26 & 25 \\
\hline $\mathrm{CV}(\%)$ & 16,3 & 20,8 & 21,2 & 9,5 & 16,2 & 13,3 & 12,1 & 13,4 & 17,8 \\
\hline
\end{tabular}

${ }^{\mathrm{ns}}$ : Não significativo $(\mathrm{P} \geq 0,05) ;{ }^{1}: \mathrm{y}=0,0566 \mathrm{~N}+10,918, \mathrm{R}^{2}=0,947(\mathrm{p}<0,01){ }^{2}: \mathrm{y}=0,1075 \mathrm{~N}$ $+17,992, \mathrm{R}^{2}=0,8979(\mathrm{p}<0,01) ;{ }^{3}: \mathrm{y}=0,1094 \mathrm{~N}+14,182, \mathrm{R}^{2}=0,9654(\mathrm{p}<0,01) ;{ }^{4}: \mathrm{y}=-$ $0,0021 \mathrm{~N}^{2}+0,2151 \mathrm{~N}+21,26, \mathrm{R}^{2}=0,8848(\mathrm{p}<0,01) ;{ }^{5}: \mathrm{y}=0,0343 \mathrm{~N}+22,800, \mathrm{R}^{2}=0,9143(\mathrm{p}$ $<0,05) ;{ }^{6}: \mathrm{y}=0,0990 \mathrm{~N}+17,864, \mathrm{R}^{2}=0,9658(\mathrm{p}<0,01)$ 
As concentrações de $\mathrm{K}$ foliar não foram influenciadas pelas fontes de $\mathrm{N}$ estudadas, já para as doses de $\mathrm{N}$, houve efeito significativo nos três cortes avaliados. No primeiro corte, a concentração de $\mathrm{K}$ foliar ajustou de modo quadrático, com teor máximo de $27 \mathrm{~g}$ de $\mathrm{K}$ $\mathrm{kg}^{-1}$ de matéria seca, na dose de $51 \mathrm{~kg}$ de $\mathrm{N}$ $\mathrm{ha}^{-1}$. Os cortes consecutivos ajustaram-se linearmente, com incremento de 3 e $8 \mathrm{~g}$ de $\mathrm{K} \mathrm{kg}^{-1}$ de matéria seca, quando compara-se testemunha com a maior dose, respectivamente, para o segundo e terceiro corte (Tabela 1).

$\mathrm{O}$ aumento na concentração de $\mathrm{N}$ foliar é explicado pelo uso das doses de $\mathrm{N}$ culminando para maior disponibilidade do nutriente no solo, nas formas de nitrato e amônio (COSTA et al., 2008). Estas formas nitrogenadas são absorvidas facilmente pelos vegetais, em vista que o $\mathrm{N}$ possui importante função nos vegetais (SOUZA \& FERNANDES, 2006).

$\mathrm{O}$ não efeito para o $\mathrm{P}$ se deve ao teor de $\mathrm{P}$ presente no solo, além da baixa necessidade de absorção do nutriente.
Entretanto, é necessário mencionar, que as áreas de forrageiras, normalmente, possuem baixos teores de $\mathrm{P}$ (quando mal manejados), necessitando maiores cuidados em sua implantação e seu no manejo. Resultado semelhante foi observado por SARTOR (2009), em avaliação da adubação nitrogenada em capim-papuã (Brachiaria plantaginea), onde apenas a não aplicação de $\mathrm{N}$ diferiu nos teores foliares de $\mathrm{P}$ das demais doses

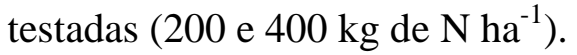

$\mathrm{O}$ aumento nas concentrações de $\mathrm{K}$ estão intimamente relacionados a alta correlação entre $\mathrm{N}$ e $\mathrm{K}$, ou seja, há sinergismo entre o $\mathrm{N}$ aplicado (e por consequência absorvido) e o $\mathrm{K}$ foliar. Esta correlação é visualizada pelos resultados da tabela 2, com grau de correlação de Pearson de 0,2550 ( $<<0,05)$, entre o N e o $\mathrm{K}$. Acredita-se que escore maior não foi encontrado, devido a dose única de aplicação de $\mathrm{K}$, sendo que, somente no primeiro corte, houve exportação de todo $\mathrm{K}$ aplicado na implantação.

Tabela 2. Correlações de Pearson entre as concentrações nutricionais, da parte aérea, do capim-xaraés, inerentes ao período experimental total (três cortes).

\begin{tabular}{|c|c|c|c|c|c|c|c|c|c|c|}
\hline Correlação & $\mathrm{N}$ & $\mathrm{P}$ & $\mathrm{K}$ & $\mathrm{Ca}$ & $\mathrm{Mg}$ & $S$ & $\mathrm{Cu}$ & $\mathrm{Fe}$ & $\mathrm{Mn}$ & $\mathrm{Zn}$ \\
\hline $\mathrm{N}$ & 1 & & & & & & & & & \\
\hline $\mathrm{P}$ & $0,427^{* *}$ & 1 & & & & & & & & \\
\hline K & $0,255^{*}$ & $-0,208^{*}$ & 1 & & & & & & & \\
\hline $\mathrm{Ca}$ & $0,724^{* *}$ & $0,472^{* *}$ & $\underset{\mathrm{ns}}{0,022}$ & 1 & & & & & & \\
\hline $\mathrm{Mg}$ & $0,599^{* *}$ & $0,588^{* *}$ & $0,{ }_{\mathrm{ns}}^{116}$ & $0,668^{* *}$ & 1 & & & & & \\
\hline S & $0,706^{* *}$ & $0,361^{* *}$ & $0, \underset{\mathrm{ns}}{195}$ & $0,578^{* *}$ & $0,500^{* *}$ & 1 & & & & \\
\hline $\mathrm{Cu}$ & $\overline{-}^{\mathrm{n}} \mathrm{n}$ & $-0,236^{*}$ & $0,317^{* *}$ & $\underset{\mathrm{ns}}{-0,008}$ & $\underset{\mathrm{ns}}{-0,109}$ & $0,237^{*}$ & 1 & & & \\
\hline $\mathrm{Fe}$ & $0,411^{* *}$ & $0,266^{*}$ & 0,182 & $0,395^{* *}$ & $0,240^{*}$ & $0,495^{* *}$ & $0,250^{*}$ & 1 & & \\
\hline Mn & 0,113 & $-\underset{n s}{-0,127}$ & $0,288^{* *}$ & 0,135 & $0,218^{*}$ & $\underset{\mathrm{ns}}{0,162}$ & $0,245^{*}$ & $0,414^{* *}$ & 1 & \\
\hline $\mathrm{Zn}$ & $0,226^{*}$ & $0,333^{* *}$ & $\underset{\mathrm{ns}}{0,083}$ & $0,324^{* *}$ & $0,336^{* *}$ & $\underset{\mathrm{ns}}{0,170}$ & $0,237^{*}$ & $\underset{\mathrm{ns}}{0,144}$ & $\underset{\mathrm{ns}}{0,095}$ & 1 \\
\hline
\end{tabular}

${ }^{\text {ns }}$ : Não significativo $(p \geq 0,05) ;{ }^{*}: p<0,05 ;{ }^{* *}: p<0,01$

A correlação entre $\mathrm{N}$ e $\mathrm{K}$ são encontrados na literatura, como Primavesi et al. (2006), que citam que as concentrações de $\mathrm{K}$ aumentaram com as 
doses de $\mathrm{N}$ aplicados no capim-marandu, da mesma forma, COSTA et al. (2008) observaram correlação positiva entre as doses de $\mathrm{N}$ aplicadas e as concentrações de $\mathrm{K}$ foliar, também PRIMAVESI et al. (2005), em capim coast-cross, observaram que as doses de $\mathrm{N}$ proporcionaram aumentos nas concentrações de $\mathrm{K}$ foliar.
Nas concentrações de macronutrientes secundários (Tabela 3), as fontes nitrogenadas estudadas não diferiram entre si, porém, nas doses avaliadas, verificou-se ajustes nas concentrações de $\mathrm{Mg}$ e $\mathrm{S}$, ambos no terceiro corte, com aumento respectivo de 0,4 e $0,3 \mathrm{~g} \mathrm{~kg}^{-1}$ (comparada a testemunha com a maior dose estudada.

Tabela 3. Concentração nutricional de cálcio, magnésio e enxofre da parte aérea do capimxaraés, em função de fontes e doses nitrogenadas em cobertura

\begin{tabular}{|c|c|c|c|c|c|c|c|c|c|}
\hline \multicolumn{10}{|c|}{ Concentração nutricional foliar $\left(\mathrm{g} \mathrm{kg}^{-1}\right)$} \\
\hline \multirow{3}{*}{ Fontes } & \multicolumn{3}{|c|}{$\mathrm{Ca}$} & \multicolumn{3}{|c|}{$\mathrm{Mg}$} & \multicolumn{3}{|c|}{$\mathrm{S}$} \\
\hline & \multicolumn{3}{|c|}{ Corte } & \multicolumn{3}{|c|}{ Corte } & \multicolumn{3}{|c|}{ Corte } \\
\hline & $1^{\mathrm{o}}$ & $2^{\circ}$ & $3^{\circ}$ & $1^{\mathrm{o}}$ & $2^{\circ}$ & $3^{\circ}$ & $1^{\circ}$ & $2^{\circ}$ & $3^{\circ}$ \\
\hline $\mathrm{U}$ & $3,0^{\mathrm{ns}}$ & $3,8^{\mathrm{ns}}$ & $3,7^{\mathrm{ns}}$ & $2,5^{\mathrm{ns}}$ & $2,7^{\mathrm{ns}}$ & $2,9^{\mathrm{ns}}$ & $1,6^{\mathrm{ns}}$ & $2,1^{\mathrm{ns}}$ & $1,8^{\mathrm{ns}}$ \\
\hline UR & 2,9 & 3,7 & 3,6 & 2,2 & 2,9 & 2,9 & 1,5 & 2,0 & 1,6 \\
\hline NA & 2,9 & 4,0 & 3,8 & 2,5 & 3,1 & 3,0 & 1,6 & 2,0 & 1,8 \\
\hline $\begin{array}{l}\text { Doses (kg } \\
\left.\mathrm{ha}^{-1}\right)\end{array}$ & $1^{\mathrm{o}}$ & $2^{\mathrm{o}}$ & $3^{\circ}$ & $1^{\mathrm{o}}$ & $2^{o}$ & $3^{\circ}$ & $1^{o}$ & $2^{o}$ & $3^{\circ}$ \\
\hline 0 & $2,8^{\mathrm{ns}}$ & $3,8^{\mathrm{ns}}$ & $3,4^{\mathrm{ns}}$ & $2,4^{\mathrm{ns}}$ & $2,9^{\mathrm{ns}}$ & $2,8^{1}$ & $1,5^{\mathrm{ns}}$ & $2,0^{\mathrm{ns}}$ & $1,6^{2}$ \\
\hline 20 & 2,8 & 3,7 & 3,6 & 2,2 & 2,7 & 2,8 & 1,6 & 2,0 & 1,7 \\
\hline 40 & 3,2 & 3,7 & 3,9 & 2,6 & 2,9 & 3,0 & 1,6 & 2,0 & 1,8 \\
\hline 80 & 3,0 & 4,2 & 3,8 & 2,4 & 3,2 & 3,1 & 1,7 & 2,1 & 1,9 \\
\hline CV (\%) & 15,0 & 12,1 & 14,0 & 18,0 & 12,9 & 8,2 & 13,6 & 7,9 & 10,2 \\
\hline
\end{tabular}

ns: Não significativo $(\mathrm{p} \geq 0,05) ;{ }^{1}: \mathrm{y}=0,0044 \mathrm{~N}+2,7766, \mathrm{R}^{2}=0,9175(\mathrm{p}<0,05) ;{ }^{2}: \mathrm{y}=$ $0,0044 \mathrm{~N}+2,7720, \mathrm{R}^{2}=0,9276(\mathrm{p}<0,05)$

De forma geral, poucos efeitos foram verificados para os macronutrientes secundários (Tabela 3). As não diferenças para as concentrações no primeiro e segundo cortes se devem a não estabilização da cultura e menor exploração radicular. Pela consequência da maior exploração e estabilização da cultura (terceiro corte) inicia-se maiores requerimentos de $\mathrm{Mg}$ e $\mathrm{S}$ pelo acúmulo de $\mathrm{N}$ foliar para a produção de compostos. Já na exportação nutricional, os efeitos no segundo corte podem estar mais correlacionados a produtividade de matéria seca que a concentração foliar dos nutrientes.

Os efeitos significativos na concentração de $\mathrm{Ca}, \mathrm{Mg}$ e $\mathrm{S}$ era esperado, devido as correlações entre os nutrientes e o N (Tabela 2), sendo, ainda, os maiores escores encontrados no presente trabalho $(0,724 ; 0,599 ;$ e 0,706 , respectivamente para $\mathrm{Ca}, \mathrm{Mg}$ e $\mathrm{S}$, em relação ao $\mathrm{N}$ ). Por conseguinte, o acúmulo de $\mathrm{N}$ foliar, perfaz maiores quantidades de $\mathrm{Ca}$, para a produção de parede celular, a fim de produção de novos compostos, tecidos e organelas. Resultados semelhantes, para a absorção de $\mathrm{Ca}$, foram encontrados por PRIMAVESI et al. (2005) e PRIMAVESI et al. (2006), com o uso de $\mathrm{N}$ na forma de ureia.

Em relação ao aumento do teor de $\mathrm{Mg}$ foliar (Tabela 3), é provável que maiores quantidades de $\mathrm{Mg}$ foram requeridas para produção de organelas, como clorofilas, estas que são responsáveis por $10 \%$ do teor total do $\mathrm{Mg}$ da folha (VITTI et al., 2006). Este resultado é confirmado por COSTA et al. (2008), onde concluíram que os teores de clorofila aumentam com as doses de N. Além da clorofila, o $\mathrm{Mg}$ acumulado pode ter sido utilizado para a ativação enzimática e estar 
presente na síntese de proteínas (VITTI et al., 2006).

Já o aumento dos teores de $\mathrm{S}$ foliar (Tabela 3), é explicado por estar intimamente ligado ao metabolismo do $\mathrm{N}$ (VITTI et al., 1988) e ser constituinte de aminoácidos (Vitti et al., 2006). BONFIMSILVA \& MONTEIRO (2006) elucidam da extrema importância de manter a relação de $\mathrm{N}$ e $\mathrm{S}$ próximo 10:1 (valores esses não longe ao do presente trabalho) para à obtenção de boa nutrição e desenvolvimento de forrageiras, culminando em máximas produções de parte aérea e melhor nutrição da forrageira.

Nas avaliações de concentração de micronutrientes não foram observadas diferenças significativa para o $\mathrm{Fe}, \mathrm{Mn}$ e $\mathrm{Zn}$, nas fontes estudadas, nos três cortes realizados, com efeito significativo para a variável $\mathrm{Cu}$, no segundo e terceiro corte (Tabela 4). Constatou-se que para o $\mathrm{Cu}$, no segundo corte, o nitrato de amônio foi $31,6 \%$ inferior a ureia e a ureia revestida, essas que não diferiram entre si, e no terceiro corte, a ureia revestida foi $33,3 \%$ inferior a ureia, sendo que, o nitrato de amônio não diferiu das duas fontes avaliadas. Como houve efeitos diferentes para cada corte, far-se-á necessário analisar a concentração de $\mathrm{Cu}$ pela média, e não por corte, para melhor discussão.

Tabela 4. Concentração nutricional de micronutrientes (cobre, ferro, manganes e zinco) da parte aérea do capim-xaraés, em função de fontes e doses nitrogenadas em cobertura

\begin{tabular}{|c|c|c|c|c|c|c|c|c|c|c|c|c|}
\hline \multicolumn{13}{|c|}{ Concentração nutricional foliar $\left(\mathrm{mg} \mathrm{kg}^{-1}\right)$} \\
\hline \multirow{3}{*}{ Fontes } & \multicolumn{3}{|c|}{$\mathrm{Cu}$} & \multicolumn{3}{|c|}{$\mathrm{Fe}$} & \multicolumn{3}{|c|}{$\mathrm{Mn}$} & \multicolumn{3}{|c|}{$\mathrm{Zn}$} \\
\hline & & Corte & & & Corte & & & Corte & & & Corte & \\
\hline & $1^{\mathrm{o}}$ & $2^{\circ}$ & $3^{\circ}$ & $1^{\mathrm{o}}$ & $2^{\circ}$ & $3^{\circ}$ & $1^{\mathrm{o}}$ & $2^{\circ}$ & $3^{\circ}$ & $1^{\mathrm{o}}$ & $2^{\circ}$ & $3^{\circ}$ \\
\hline $\mathrm{U}$ & $17^{\mathrm{ns}}$ & $19 \mathrm{a}$ & $15 \mathrm{a}$ & $548^{\mathrm{ns}}$ & $\underset{\mathrm{ns}}{1.580}$ & $698^{\mathrm{ns}}$ & $\begin{array}{c}109 \\
\text { ns }\end{array}$ & $\begin{array}{c}107 \\
\text { ns }\end{array}$ & $96^{\mathrm{ns}}$ & $32^{\mathrm{ns}}$ & $30^{\mathrm{ns}}$ & $32^{\mathrm{ns}}$ \\
\hline UR & 19 & $19 \mathrm{a}$ & $10 \mathrm{~b}$ & 485 & 1.486 & 784 & 102 & 110 & 106 & 28 & 30 & 31 \\
\hline NA & 17 & $13 \mathrm{~b}$ & $\begin{array}{l}13 \\
a b\end{array}$ & 482 & 1.321 & 859 & 106 & 111 & 102 & 29 & 30 & 33 \\
\hline
\end{tabular}

\begin{tabular}{|c|c|c|c|c|c|c|c|c|c|c|c|c|}
\hline $\begin{array}{l}\text { Doses } \\
\left(\begin{array}{c}\mathrm{kg} \mathrm{ha}^{-} \\
1\end{array}\right)\end{array}$ & $1^{o}$ & $2^{o}$ & $3^{\circ}$ & $1^{o}$ & $2^{o}$ & $3^{\circ}$ & $1^{\mathrm{o}}$ & $2^{o}$ & $3^{\circ}$ & $1^{o}$ & $2^{\circ}$ & $3^{\circ}$ \\
\hline 0 & $17^{\mathrm{ns}}$ & $14^{\mathrm{ns}}$ & $15^{\mathrm{ns}}$ & $475^{\mathrm{ns}}$ & $\underset{\mathrm{ns}}{1.722}$ & $690^{1}$ & $\underset{\mathrm{ns}}{100}$ & $\begin{array}{c}109 \\
\text { ns }\end{array}$ & $95^{2}$ & $27^{3}$ & $28^{\mathrm{ns}}$ & $29^{\mathrm{ns}}$ \\
\hline 20 & 17 & 18 & 14 & 481 & 1.383 & 729 & 100 & 113 & 98 & 27 & 30 & 29 \\
\hline 40 & 22 & 24 & 14 & 478 & 1.356 & 776 & 110 & 108 & 108 & 30 & 29 & 33 \\
\hline 80 & 16 & 21 & 14 & 586 & 1.387 & 927 & 112 & 106 & 104 & 34 & 32 & 35 \\
\hline $\begin{array}{l}\mathrm{CV} \\
(\%)\end{array}$ & 32,5 & 31,0 & 34,8 & 31,8 & 38,6 & $22,1^{\#}$ & 14,4 & 19,0 & 19,7 & 14,8 & 13,2 & 24,0 \\
\hline
\end{tabular}

\#: Dados transformados $(\mathrm{x}+0,5)^{0,5} ;{ }^{\mathrm{ns}}$ : Não significativo $(\mathrm{p} \geq 0,05) ;{ }^{1}: \mathrm{y}=6,576 \mathrm{~N}+724,85$, $\mathrm{R}^{2}=0,9763(\mathrm{p} \geq 0,05) ;{ }^{2}: \mathrm{y}=-0,0128 \mathrm{~N}^{2}+1,5741 \mathrm{~N}+95,111, \mathrm{R}^{2}=0,9999(\mathrm{p}<0,05) ;{ }^{3}: \mathrm{y}=$ $0,0361 \mathrm{~N}+25,135, \mathrm{R}^{2}=0,974(\mathrm{p}<0,01$

Nas doses de $\mathrm{N}$ avaliadas, as concentrações nutricionais ajustaram-se, no primeiro corte, o $\mathrm{Zn}$; no terceiro corte, o $\mathrm{Fe}$ e o $\mathrm{Mn}$, com o $\mathrm{Zn}$ e $\mathrm{Fe}$, ajustando linearmente e o Mn de modo quadrático (Tabela 4). Obtendo incrementos nas concentrações, respectivamente para o $\mathrm{Zn}$ e Fe, de 7 e $237 \mathrm{~g} \mathrm{~kg}^{-1}$, em comparação à testemunha com a maior dose de $\mathrm{N}$, e para o Mn houve aumento de 48,5 $\mathrm{g} \mathrm{kg}^{-1}$ em comparação da dose de máxima eficiência $\left(61,5 \mathrm{~kg} \mathrm{ha}^{-1}\right.$ de N) com $0 \mathrm{~kg} \mathrm{ha}^{-1}$.

Os efeitos verificados na concentração de Fe é devido a correlação existente entre o Fe e $\mathrm{N}$ (Tabela 2; escore de $0,411(\mathrm{p}<0,01))$, ou seja, o aumento de 
$\mathrm{N}$ na planta faz necessárias maiores quantidades de $\mathrm{Fe}$, em vista das funções do Fe no vegetal, como: ativação enzimática, participar de reações fundamentais de oxirredução; como na ferrodoxina e enzimas redutase; nitrogenase e sulfato redutase (DECHEN \& NACHTIGALL, 2006). Inclusive, como a ferrodoxina é uma proteína que possui $\mathrm{Fe}$ e $\mathrm{S}$, verifica-se também, alta correlação entre o $\mathrm{Fe}$ e $\mathrm{S}$ (escore $0,495(\mathrm{p}<0,01))$.

No Mn, apesar da não significância na correlação com o N (Tabela 2), podia-se esperar pela correlação, em vista que o Mn tem ativa participação na fotossíntese e na síntese de clorofila. A não correlação pode ser explicado pelo o uso de dados de todo período experimental e não por corte, na correlação. Quando analisado a correlação de $\mathrm{N}$ com $\mathrm{Mn}$ por corte, observa-se os seguintes escores: 0,066; -0,126; 0,458 (respectivamente para o primeiro, segundo e terceiro cortes), sendo que, somente no terceiro corte houve correção significativa ( $p<0,05$ ), esses dados confirma a hipótese levantada, assim, o ajuste para o Mn no terceiro corte, é devido a estabilização da cultura somada a correlação com o $\mathrm{N}$.

$\mathrm{O}$ teor de $\mathrm{Cu}$ foliar não diferiu entre as doses de $\mathrm{N}$ estudadas (Tabela 4), porém, nota-se altos teores foliares, em relação aos adequados para a cultura (4-12 $\mathrm{mg} \mathrm{kg}^{-1}$ ) (RAIJ et al., 1997), independentemente dos tratamentos avaliados. Estes altos teores podem ser explicados pela mineralização da matéria orgânica do solo (MOS) oriunda das culturas antecessoras, ocasionando maior disponibilidade e consequentemente maior absorção do $\mathrm{Cu}$ (ABREU et al., 2001), já que, grande parte do $\mathrm{Cu}$ está adsorvido ou imobilizado pela MOS.

O efeito verificado na concentração de Zn (Tabela 4) se deve a sua participação no metabolismo da planta no crescimento vegetal, afetando a síntese e conservação de hormônios (DECHEN E NACHTIGALL, 2006) como, por exemplo, na síntese de auxina (CAKMAk et al., 1989), este, com papel no alongamento, dominância apical e formação de raízes (TAIZ \& ZEIGER, 2006). É possível que a pastagem, em sua implantação, necessitou maiores quantidades de $\mathrm{Zn}$, para melhor desenvolvimento e estabilização. A maior absorção de $\mathrm{Zn}$ pela planta com a aplicação de $\mathrm{N}$ foi detectada por LANGIN et al. (1962) e SOLTAPOUR (1969). Já COSTA et al. (2009), em avaliação de capim-marandu, encontraram efeito de diluição, porém, mesmo com o efeito diluição, maiores quantidades de $\mathrm{Zn}$ foram absorvidas.

Quando avaliada a concentração média nutricional, observou-se que para as fontes avaliadas, não houve diferença para o N, P, K, Ca, Mg, S, Fe Mn e Zn (Tabela 5). Já nas concentrações de $\mathrm{Cu}$, houve efeito significativos para as fontes avaliadas, com a ureia superior ao nitrato de amônio, estes que não diferiram para a ureia revestida. 
Tabela 5. Concentração nutricional média de macronutrientes e micronutrientes de parte aérea do capim-xaraés, inerente ao período de implantação da cultura, em função de fontes e doses nitrogenadas

\begin{tabular}{|c|c|c|c|c|c|c|c|c|c|c|}
\hline \multicolumn{11}{|c|}{ Concentração média nutricional } \\
\hline Fontes & $\mathrm{N}$ & $\mathrm{P}$ & $\begin{array}{c}\mathrm{K} \\
\mathrm{g} \mathrm{kg}^{-1}\end{array}$ & $\mathrm{Ca}$ & $\mathrm{Mg}$ & $\mathrm{S}$ & $\mathrm{Cu}$ & $\begin{array}{c}\mathrm{Fe} \\
\mathrm{mg} \mathrm{kg}^{-1}\end{array}$ & $\mathrm{Mn}$ & $\mathrm{Zn}$ \\
\hline $\mathrm{U}$ & $\underset{\mathrm{ns}}{17,5}$ & $3,1^{\mathrm{ns}}$ & $\underset{\mathrm{ns}}{23,1}$ & $3,5^{\mathrm{ns}}$ & $2,7^{\mathrm{ns}}$ & $1,8^{\mathrm{ns}}$ & $\begin{array}{c}17,9 \\
\mathrm{a}\end{array}$ & $\begin{array}{c}1.006 \\
\mathrm{~ns}\end{array}$ & $99^{\mathrm{ns}}$ & $33^{\mathrm{ns}}$ \\
\hline UR & 16,7 & 3,2 & 23,7 & 3,4 & 2,7 & 1,7 & $\begin{array}{c}15,3 \\
a b\end{array}$ & 917 & 101 & 30 \\
\hline NA & 18,7 & 3,1 & 23,5 & 3,6 & 2,9 & 1,8 & $\begin{array}{c}14,7 \\
\mathrm{~b}\end{array}$ & 860 & 98 & 31 \\
\hline $\begin{array}{c}\text { Doses (kg } \\
\left.\mathrm{ha}^{-1}\right)\end{array}$ & $\mathrm{N}$ & $\mathrm{P}$ & $\begin{array}{c}\mathrm{K} \\
\mathrm{g} \mathrm{kg}^{-1}\end{array}$ & $\mathrm{Ca}$ & $\mathrm{Mg}$ & $\mathrm{S}$ & $\mathrm{Cu}$ & $\begin{array}{c}\mathrm{Fe} \\
\mathrm{mg} \mathrm{kg}^{-1}\end{array}$ & $\mathrm{Mn}$ & $\mathrm{Zn}$ \\
\hline 0 & $14,4^{1}$ & $3,3^{\mathrm{ns}}$ & $20,8^{2}$ & $3,3^{3}$ & $2,7^{\mathrm{ns}}$ & $1,7^{4}$ & $15^{\mathrm{ns}}$ & $908^{\mathrm{ns}}$ & $97^{\mathrm{ns}}$ & $30^{5}$ \\
\hline 60 & 16,6 & 3,2 & 22,3 & 3,3 & 2,6 & 1,8 & 15 & 908 & 94 & 30 \\
\hline 120 & 17,8 & 2,9 & 25,2 & 3,6 & 2,8 & 1,8 & 19 & 875 & 105 & 31 \\
\hline 240 & 21,7 & 3,1 & 25,4 & 3,7 & 2,9 & 1,9 & 15 & 1.022 & 103 & 34 \\
\hline $\mathrm{CV}(\%)$ & 17,3 & 8,8 & 10,2 & 9,1 & 9,9 & 7,6 & 18,8 & 24,3 & 8,8 & 10,9 \\
\hline
\end{tabular}

${ }^{\mathrm{n}}$ : Não significativo $(\mathrm{p} \geq 0,05) ;{ }^{1}:{ }^{\mathrm{y}}=0,0301 \mathrm{~N}+14,458, \mathrm{R}^{2}=0,9929(\mathrm{p}<0,01) ;{ }^{2}: \mathrm{y}=-$ $0,0001 \mathrm{~N}^{2}+0,0481 \mathrm{~N}+20,525, \mathrm{R}^{2}=0,9495(\mathrm{p}<0,01) ;{ }^{3}: \mathrm{y}=0,0015 \mathrm{~N}+3,3323, \mathrm{R}^{2}=0,7942$ $(\mathrm{p}<0,05) ;{ }^{4}: \mathrm{y}=0,0008 \mathrm{x}+1,72, \mathrm{R}^{2}=0,9143(\mathrm{p}<0,05) ;{ }^{5}: \mathrm{y}=0,0166 \mathrm{~N}+29,566, \mathrm{R}^{2}=$ $0,8890(\mathrm{p}<0,05)$

A diferença observada na concentração foliar de $\mathrm{Cu}$, para as fontes de $\mathrm{N}$ avaliadas, pode estar correlacionada com a acidificação do solo pelos fertilizantes nitrogenados (LEAL et al., 2007; COSER et al., 2007). Por consequência, da acidificação do solo, maior quantidade de $\mathrm{Cu}$ é disponibilizada na solução, tornando-se passível de absorção (ABREU et al., 2006).

Em avaliação das doses nitrogenadas, observa-se não ajuste na concentração de N, K, Ca, S e Zn (Tabela 5). Na concentração média nutricional, o $\mathrm{N}, \mathrm{Ca}, \mathrm{S}$ e $\mathrm{Zn}$ ajustaram linearmente, com aumentos respectivos de 7,3, 0,4 e 0,2 $\mathrm{g}$ $\mathrm{kg}^{1}$ e $4 \mathrm{mg} \mathrm{kg}^{-1}$. A concentração de $\mathrm{K}$ ajustou de modo quadrático, com concentração máxima $\left(26,3 \mathrm{~g} \mathrm{~kg}^{-1}\right)$ na dose de $240,5 \mathrm{~kg} \mathrm{ha}^{-1}$ de N.

Inerente aos resultados nota-se que o $\mathrm{N}$ aplicado culmina no aumento de $\mathrm{K}, \mathrm{Ca}$, $\mathrm{S}$ e $\mathrm{Zn}$, de modo geral, assim, é possível que esses nutrientes possam ser mais requisitados na implantação da pastagem de capim-xaraés, fazendo-se necessários maiores cuidados, para não se tornarem limitantes à produtividade e para boa formação de pastagem. Porém, destaca que mesmo os demais nutrientes avaliados não terem sido influenciados pelos tratamentos, não os tornam menos importantes, e, não se deve abster da manutenção dos mesmos. Ainda, é notório, que o capim-xaraés é mais responsivo que a dose máxima avaliada (80 kg ha-1 de $\mathrm{N}$ ), já que em algumas variáveis, as concentrações ajustaram de modo linear, e algumas quadráticas, com o máximo após a dose máxima de $\mathrm{N}\left(80 \mathrm{~kg} \mathrm{ha}^{-1} \mathrm{de} \mathrm{N}\right)$.

Não foi verificada diferença entre as fontes estudadas, exceto para a concentração de $\mathrm{Cu}$ foliar, apesar de estudos demonstrarem que o nitrato de amônio tende à menor perda por volatilização e a ureia revestida ter como princípio a maior eficiência (BERNARDI et al., 2010), quando comparada com a ureia convencional. Vale ressaltar que a adubação de cobertura foi realizada a lanço e sem incorporação, o que pode ocasionar maior perda da ureia por volatilização (SANGOI et al., 2003). Entretanto, perdas de $\mathrm{N}$ já são previsíveis, e é pouco 
frequente que seja transposto à produtividade (LARA CABEZAS et al., 1997). Além disto, vale destacar que o solo úmido, favoreceu na difusão da ureia convencional para o interior do solo, nivelando, deste modo, o efeito de todas as fontes estudadas.

Quando analisado dados inerentes a fertilizantes de alta eficiência (ureia revestida), há escassez de resultados para pastagens, sendo mais comumente encontrados para grandes culturas, como o feijão (VALDERRAMA et al., 2009), o milho (GUARESCHI et al., 2011; VALDERRAMA et al., 2011; VALDERRAMA et al., 2014) e algodão (SANTINI et al., 2013), tornando assim, de difícil comparação com os presentes dados.

$\mathrm{O}$ não efeito dos fertilizantes revestidos é estudado por ZAVASCHI (2010), onde o autor conclui que a aplicação de ureia revestida por polímeros não reduz as perdas por volatilização e não influencia nos teores de amônio e nitrato do solo, quando comparada com a ureia convencional. Fato também estudado por TASCA et al. (2011), onde concluíram que o uso de inibidores não propicia menor perda de $\mathrm{N}$, ocasionando apenas atraso no pico de volatilização.

Por outro lado, deve-se levar em consideração que estudos desta natureza nem sempre são adequadas para avaliar à eficiência das fontes, principalmente, em pesquisas de curto prazo, pois a maior parte do nitrogênio absorvido pelas plantas provém do solo (DOBERMANN, 2005). De acordo com CANTARELLA \& MONTEZANO (2010), em muitas situações, a ausência de redução de produção pode não significar necessariamente ausência de perdas de $\mathrm{NH}_{3}$.

Quando analisados os trabalhos supracitados, nota-se que poucas respostas são encontradas para fertilizantes de alta eficiência, não tendo dados suficientes para comprovar sua maior eficácia, fazendo-se que o uso dessa tecnologia seja desnecessário e apenas gasto monetário.

Em avaliação das concentrações nutricionais foliares do capim-xaraés, o macronutriente com maior concentração é o K (Tabela 6). Ordenando a concentração foliar, posterior ao $\mathrm{K}$, segue $\mathrm{O}$ $\mathrm{N}>\mathrm{Ca}>\mathrm{P}>\mathrm{Mg}>\mathrm{S}$, já os micronutrientes, $\mathrm{Fe}>\mathrm{Mn}>\mathrm{Zn}>\mathrm{Cu}$.

Tabela 6. Concentração nutricional da parte área do capim-xaraés, inerentes a todo período experimental (três cortes), com seus respectivos valores médios, máximos e mínimos (de acordo com intervalo de confiança, usando a distribuição T de Student a 5\%), desvio padrão $\operatorname{amostral}(\sigma)$ e coeficiente de variação $(\mathrm{CV})$

\begin{tabular}{cccccc}
\hline \multirow{2}{*}{ Nutrientes } & \multicolumn{5}{c}{ Concentração nutricional } \\
\cline { 2 - 6 } & Média $^{*}$ & Máxima & Mínima & $\sigma$ & $\mathrm{CV}(\%)$ \\
\cline { 2 - 6 } $\mathrm{N}$ & $17,6 \mathrm{~b}$ & 18,8 & 16,4 & 5,2 & 29,3 \\
$\mathrm{P}$ & $3,1 \mathrm{~d}$ & 3,3 & 3,0 & 0,8 & 24,6 \\
$\mathrm{~K}$ & $23,4 \mathrm{a}$ & 24,3 & 22,6 & 3,6 & 15,5 \\
$\mathrm{Ca}$ & $3,5 \mathrm{c}$ & 3,6 & 3,3 & 0,6 & 17,8 \\
$\mathrm{Mg}$ & $2,7 \mathrm{e}$ & 2,8 & 2,6 & 0,4 & 15,9 \\
$\mathrm{~S}$ & $1,8 \mathrm{f}$ & 1,8 & 1,7 & 0,3 & 14,8 \\
\hline Nutrientes & $15,9 \mathrm{~d}$ & 17,3 & 14,7 & 5,6 & 35,0 \\
\hline $\mathrm{Cu}$ & $928,4 \mathrm{a}$ & 1049,6 & 807,1 & 516,0 & 55,6 \\
$\mathrm{Fe}$ & $99,7 \mathrm{~b}$ & 103,9 & 95,5 & 18,0 & 18,1 \\
$\mathrm{Mn}$ & $31,3 \mathrm{c}$ & 32,7 & 29,9 & 6,0 & 19,2 \\
\hline $\mathrm{Zn}$ & & \multicolumn{5}{c}{ Micronutrientes $\left(\mathrm{mg} \mathrm{kg}^{-1}\right)$} \\
\hline
\end{tabular}

*. Médias seguidas pelas mesmas (entre macro e micronutrientes) letras na coluna, não difere no nível 0,05 , de acordo com teste de Wilcoxon. 
A ordem de concentração nutricional aproxima ao encontrado por COSTA et al. (2009), citando o K como o macronutriente e o $\mathrm{Fe}$ como o micronutriente mais extraído pela Urochloa brizantha cv. Marandú. Nas concentrações de nutrientes foliares encontrados no presente trabalho, quando comparados com os teores adequados, citados por RAIJ et al. (1997), nota-se que o $\mathrm{P}, \mathrm{Cu}$ e $\mathrm{Fe}$ estão acima do adequado, para gramíneas do Grupo II (Tabela 6). A concentração de $\mathrm{P}$, é inteiramente ligada ao teor de $\mathrm{P}$, inicial, na área (29 $\left.\mathrm{mg} \mathrm{dm}^{-3}\right)$, mas, salienta-se, que a maioria dos solos com pastagens,

\section{CONCLUSÕES}

A ureia convencional é a recomendada para a implantação de Urochloa brizantha cv. Xaraés, quando comparado à ureia revestida por polímeros e ao nitrato de amônio, em vista que as mesmas não diferiram, e, a ureia convencional, é a de menor custo por quilograma de $\mathrm{N}$.

\section{REFERÊNCIAS}

ABREU, E. M. A.; FERNANDES, A. R.; MARTINS, A. R. A.; RODRIGUES, T. E. Produção de forragem e valor nutritivo de espécies forrageiras sob condições de pastejo, em solo de várzea baixa do Rio Guamá. Acta Amazônica,, v. 36(1): 1118, 2006. Disponível em <https://www.embrapa.br/amazoniaoriental/busca-de-publicacoes//publicacao/577045/producao-deforragem-e-valor-nutritivo-de-especiesforrageiras-sob-condicoes-de-pastejo-emsolo-de-varzea-baixa-do-rio-guama>

BERNARDI, A. C. C.; MOTA, E. P.; CARDOSO, R. D.; OLIVEIRA, P. P. A. Volatilização de amônia, produção de matéria seca e teores foliares de $\mathbf{N}$ do azevém adubado com fontes nitrogenadas. São Carlos, p.8, 2010. (Circular técnica, 66). Disponível em <http://www.cppse.embrapa.br/volatiliza- principalmente mal manejadas, apresentam níveis críticos de $\mathrm{P}$, necessitando assim, maiores cuidados na adubação fosfatada na implantação de forrageiras.

As altas concentrações de $\mathrm{Cu}$ são explicadas pela consequência da mineralização da MOS, proveniente das culturas antecessoras, o que, pela ocasião, resultaram em maior disponibilidade e consequentemente torna-se possível maior absorção de Cu (ABREU et al., 2006). Já as concentrações de $\mathrm{Fe}$, bem como de $\mathrm{Cu}$, podem caracterizar o capim-xaraés como cultura de tolerância às altas concentrações de $\mathrm{Fe}$ e $\mathrm{Cu}$.

A ureia revestida não foi caracterizada como fertilizante de alta eficiência.

A dose de nitrogênio recomendada é de $80 \mathrm{~kg} \mathrm{ha}^{-1}$ por corte, na implantação do capim-xaraés, devido ao aumento nutricional da forrageira. O uso de doses $\mathrm{N}$ culmina no aumento da concentração de $\mathrm{N}$ foliar.

o-de-am-nia-produ-o-de-mat-ria-seca-eteores-foliares-de-n-do-azev-m-adubadocom-fontes-n >

BONFIM-SILVA, E. M.; MONTEIRO, F. A. Nitrogênio e enxofre em características produtivas do capim-braquiária proveniente de área de pastagem em degradação. Revista Brasileira de Zootecnia, v. 35(4): 1289-1297, 2006. Disponível em <http://www.scielo.br/scielo.php?script=sc i_arttext\&pid=S151635982006000500006>

CABRAL, W. B.; SOUZA, A. L.; ALEXANDRINO, E.; TORAL, F. L. B.; SANTOS, J. N.; CARVALHO, M. V. P. Características estruturais e agronômicas da Brachiariabrizantha cv. Xaraés submetida a doses de nitrogênio. Revista Brasileira de Zootecnia, v.41(4): 846855, 2012. Disponível em 
<http://www.scielo.br/pdf/rbz/v41n4/04.pd f $>$

CAKMAK, K.; MARSCHNER, H.; BANGERTH, F. Effect of zinc nutritional status on growth, protein metabolism and levels of indole-3-acetic acid and other phytohormones in bean (Phaseolus vulgaris L.). Journal of Experimental Botany, v.40: 405-412, 1989. Disponível em

$<$ http://jxb.oxfordjournals.org/content/40/3 |405>

CANTARELLA, H.; MONTEZANO, Z. F. Nitrogênio e enxofre. In: PROCHNOW, L. I.; CASARIN, V.; STIPP, S. R. (Ed.). Boas práticas para uso eficiente de fertilizantes: nutrientes. Piracicaba: International Plant Nutrition Institute, 2010. p. 5-46.

COSER, T. R.; RAMOS, M. L. G.; AMABILE, R. F.; RIBEIRO JR, W. Q. Nitrogênio da biomassa microbiana em solo de Cerrado com aplicação de fertilizante nitrogenado. Pesquisa agropecuária brasileira, v.42(3): 399406, 2007. Disponível em $<$ http://www.scielo.br/scielo.php?script=sc i_arttext\&pid=S0100-

204X2007000300014>

COSTA, K. A. P.; FAQUIN, V.; OLIVEIRA, I. P.; ARAÚJO, J. L.; RODRIGUES, R. B. Doses e fontes de nitrogênio em pastagem de capimmarandu. II - Nutrição nitrogenada da planta. Revista Brasileira de Ciência do Solo, v.32: 1601-1607, 2008. Disponível em

$<$ http://www.scielo.br/scielo.php?script=sc i arttext\&pid=S010006832008000400024>

COSTA, K. A. P.; FAQUIN, V.; OLIVEIRA, I. P.; SEVERIANO, E. C.; SIMON, G. A.; CARRIJO, M. S. Extração de nutrientes do capim-marandu sob doses e fontes de nitrogênio. Revista Brasileira de Saúde e Produção Animal, v.10(4): 801-812, 2009.

COSTA, K. A. P.; SEVERIANO, E. S. C.; SILVA, F. G.; BORGES, E. F.;
EPIFÂNIO, P. S.; GUIMARÃES, K. C. Doses and sources of nitrogen on yield and bromatological composition of Xaraés grass. Ciência Animal Brasileira, v.14(3): 288-298, 2013. Disponível em <http://www.scielo.br/scielo.php?script=sc i_arttext\&pid=S1809$\underline{68912013000300003>}$

DECHEN， R.A.; NACHTIGALL， G.R. Micronutrientes. In: FERNANDES, M.S (ed). Nutrição mineral de plantas. Viçosa, MG, do Sociedade Brasileira de Ciência Solo, 2006. p.327-354.

DOBERMANN, A. Nitrogen use efficiency - state of the art. In: INTERNATIONAL WORKSHOP ON ENHANCED-EFFICIENCY

FERTILIZERS, 2005, Frankfurt. Proceedings... Paris: International Fertilizer Industry Association, 2005.

EMBRAPA. Sistema brasileiro de classificação de solo. 3. ed. Rio de Janeiro: Centro Nacional de Pesquisa de Solos, 2013. 353 p.

FAGUNDES, J.L.; FONSECA, D.M.; MORAIS, R.V.; MISTURA, C.; VITOR, C.M.T.; GOMIDE, J.A.; NASCIMENTO JUNIOR, D.; SANTOS, M.E.R. \& LAMBERTUCCI, D.M. Avaliação das características estruturais do capimbraquiária em pastagens adubadas com nitrogênio nas quatro estações do ano. Revista Brasileira de Zootecnia, v.35(1): 30-37, 2006.

FERREIRA, D. F. Programa de análises estatísticas (Statistical Analysis Software) e planejamento de experimentos. Lavras: Universidade Federal de Lavras, 2003. Software.

GUARESCHI, R. F.; GAZOLLA, P. R.; PERIN, A. SANTINI, J. M. K. Adubação antecipada da cultura da soja com superfosfato triplo e cloreto de potássio revestidos por polímeros. Ciência e Agrotecnologia, v. 35(4): 643-648, 2011. Disponível em <http://www.scielo.br/scielo.php?script=sc i_arttext\&pid=S141370542011000400001> 
HOFFMANN, A.; MORAES, E. H. B. K.; MOUSQUER, C. J.; SIMIONI, T. A.; JUNIOR GOMES, F.; FERREIRA, V. B.; SILVA, H. M. Produção de bovinos de corte no sistema de pasto-suplemento no período seco. Nativa, v. 02(2): 119-130, 2014.

IBGE - Instituto Brasileiro de Geografia e Estatística. IBGE. Censo agropecuário 2006: dados preliminares. Rio de Janeiro, p.1-146, 2006.

LANGIN, E. J.; WARD, R. C.; OLSON, R. A.; RHOADES, H. F. Factors responsible for poor response of corn and grain sorghum to phosphorus fertilization: II. Lime and P placement effects on P-Zn relations. Soil Science Society of America Proceedings. v.26: 574-578, 1962. Disponível em <https://dl.sciencesocieties.org/publication s/sssaj/abstracts/26/6/SS0260060574?acces $\mathrm{s}=0 \&$ view $=\mathrm{pdf}>$

LARA CABEZAS, W. A. R.; KORNDORFER, G. H.; MOTTA. S. A. Volatilização de $\mathrm{N}^{-\mathrm{NH}_{3}}$ na cultura de milho: II. Avaliação de fontes sólidas e fluidas em sistema de plantio direto e convencional. Revista Brasileira de Ciência do solo, v.21(3): 489-496, 1997. Disponível em $<$ http://www.scielo.br/pdf/rbcs/v21n3/19.p df $>$

LEAL, R. M.; NATALE, W.; RENATO DE MELLO PRADO, R. M.; ZACCARO, R. P. Adubação nitrogenada na implantação e na formação de pomares de caramboleira. Pesquisa agropecuária brasileira, v.42(8): p.1111-1119, 2007. Disponível em <http://www.scielo.br/scielo.php?script=sc i_arttext\&pid=S0100204X2007000800007>

MALAVOLTA, E.; VITTI, G. C.; OLIVEIRA, S.A. Avaliação do estado nutricional das plantas: princípios e aplicações. $\quad 2$.ed. Piracicaba: POTAFOS, 1997. 319p.

PRIMAVESI, A.C.; PRIMAVESI, O.; CORRÊA; L. A.; CANTARELLA, H.;
SILVA, A. G. Absorção de cátions e ânions pelo capim-coastcross adubado com ureia e nitrato de amônio. Pesquisa Agropecuária Brasileira, v.40(3): 247253, 2005. Disponível em <http://www.scielo.br/scielo.php?script=sc i arttext\&pid=S0100-

204X2005000300008>

PRIMAVESI, A. C.; PRIMAVESI, O.; CORRÊA, L. A.; SILVA, A. G.; CANTARELLA, H. Nutrientes na fitomassa de capim-marandu em função de fontes e doses de nitrogênio. Ciência e Agrotecnologia, v.30(3): 562-568, 2006. Disponível

em <http://www.scielo.br/scielo.php?script=sc i_arttext\&pid=S1413-

70542006000300024>

RAIJ, B. van; CANTARELLA, H.; QUAGGIO, J. A.; FURLANI, A. M. C. Recomendações de calagem e adubação para o Estado de São Paulo. Campinas: Instituto Agronômico de Campinas, 1997, 285p. (Boletim técnico, 100)

SANGOI, L.; ERNANI, P. R.; LECH, V. A.; RAMPAZZO, C. Volatilização de N$\mathrm{NH}_{3}$ em decorrência da forma de aplicação de uréia, manejo de resíduos e tipo de solo, em laboratório. Ciência Rural, v.33(4): 687-692, 2003. Disponível em <http://www.scielo.br/scielo.php?script=sc i_arttext\&pid=S0103$\underline{84782003000400016>}$

SANTINI, J. M. K. PERIN, A.; SILVEIRA, F. O.; LOPES FILHO, L. C.; VALDERRAMA, M. The usage of NPK coated by polymers on the cotton crop (Gossypium hirsutum 1.) for biomass production on the aerial part. Global Science and Technology, v. 6(3): 79-89, $2013 . \quad$ Disponível em <http://rv.ifgoiano.edu.br/periodicos/index. $\mathrm{php/gst/article/view/608>}$

SARTOR, L. R. Eficiência de utilização de nitrogênio, fosforo e potássio por plantas de Papuã submetidas a diferentes intensidades de pastejo e níveis de nitrogênio. Pato Branco: UTFPR, 2009. 115p. Dissertação 
(Mestrado) - Programa de Pós-graduação em Agronomia. Pato Branco. Universidade Tecnológica Federal do Paraná. Pato Branco, 2009. Disponível em $<$ http://bdtd.ibict.br/vufind/Record/UTPR 8058c0b9bd8955d57596c322eb1dfcc5 >

SOLTAPOUR, P. N. Effect of nitrogen, phosphorus, and zinc placement on yield and composition of potatoes. Agronomic journal, v.61: 288-289, 1969.

SOUZA, S. R.; FERNANDES, M. S. Nitrogênio. In: FERNANDES, M. S. Nutrição Mineral de Plantas. Viçosa MG. Editora Sociedade Brasileira de Ciência do Solo, 2006. Cap. 9. p. 216-252. Disponível em $<$ https://dl.sciencesocieties.org/publication s/aj/abstracts/61/2/AJ0610020288?access= $\underline{0 \& \text { view }=\text { pdf }>}$

TAIZ, L.; ZEIGER, E. Fisiologia Vegetal.3. ed. São Paulo: Artmed, 2006.

TASCA, F. A.; ERNANI. P. R.; ROGERI, D. A.; GATIBONI, L. P.; CASSOL, P. C. Volatilização de amônia do solo após a aplicação de ureia convencional ou com inibidor de uréase. Revista Brasileira de Ciência do Solo, v.35: 493-502, 2011. Disponível em $<$ http://www.scielo.br/pdf/rbcs/v35n2/v35n 2a18.pdf $>$

USDA - United States Department of Agriculture. Livestock and Poultry: World Markets and Trade. Foreign Agricultural Service, 2014.

VALDERRAMA, M.; BUZETTI, S.; BENETT, C. G. S.; ANDREOTTI, M.; ARF, O.; SÁ, M. E. Fontes e doses de nitrogênio e fósforo em feijoeiro no sistema plantio direto. PesquisaAgropecuária Tropical, v. 39(3): 191-196, 2009.

VALDERRAMA, M.; BUZETTI, S.; BENETT, C. G. S.; ANDREOTTI, M.; TEIXEIRA FILHO, M. C. M. Fontes e doses de NPK em milho irrigado sob plantio direto. Pesquisa Agropecuária Tropical, v. 41(2): 254-263, 2011. Disponível
$<$ http://www.scielo.br/pdf/pat/v41n2/a15.p df $>$

VALDERRAMA, M.; BUZETTI, S.; TEIXEIRA FILHO, M. C. M.; BENETT, C. G. S.; ANDREOTTI, M. Adubação nitrogenada na cultura do milho com ureia revestida por diferentes fontes de polímeros. Semina: Ciências Agrárias, v. 35(2): 659-670, 2014. Disponível em <http://www.uel.br/revistas/uel/index.php/ semagrarias/article/view/13168 >

VIEIRA, B.A.R.M.; TEIXEIRA, M.M. Adubação de liberação controlada chega como solução. Revista Campo \& Negócios, v.3(41): 04-08, 2004.

VITTI, G.C. et al. Resposta de culturas anuais e perenes à aplicação de enxofre. In: BORKERT, C.M. (ed). Enxofre e micronutrientes na agricultura brasileira. Londrina: Embrapa Soja/IAPAR/SBCS, 1988. p.61-85.

ZAVASCHI, E. Volatilização de amônia e produtividade do milho em função da aplicação de ureia revestida com polímeros. Piracicaba: ESALQ/USP. 2010. 92p. Dissertação (Mestrado) Programa de Pós-graduação em Ciências - Solos e Nutrição de Plantas. Escola Superior de Agricultura Luiz de Queiroz, Universidade de São Paulo, Piracicaba, 2010. Disponível em <http://www.teses.usp.br/teses/disponiveis/ 11/11140/tde-17092010-172528/pt-br.php 